\title{
Nonlinear Dynamic Coupling Analysis on Gear System of Automated Mechanical Transmission ${ }^{1}$

\author{
Qiang SONG ${ }^{a *}$, Ping-Yang SUI
}

School of Mechanical Engineering, Beijing Institute of Technology, Beijing, 100081, China

asongqiang@bit.edu.cn, b530320766@qq.com

${ }^{*}$ Corresponding author

Keywords: mode superposition method, FFT spectrum, Poincare maps, nonlinear dynamic.

\begin{abstract}
In the process of the automated mechanical transmission operating, one pair of cogwheels are engaged with the other idling. Because both gear pairs are supported on one shaft, the states of them will be mutual coupling. By analyzing the meshing stiffness, mesh damp, support stiffness and coupling state via mode superposition method, the five dimension degree model of automated mechanical transmission has been built and compared with the model which neglects the coupling impact. Simulated in MATLAB environment and calculation of the state response of nonlinear dynamic systems by presenting long-time histories, spectra of the response, poincare maps and FFT spectrum. The results are compared and show the coupling factor will influence the response of the gear system.
\end{abstract}

\section{Introduction}

The gear drive system is widely used in automotive transmission. Due to the existence of the tooth backlash, the support gap and the time-varying stiffness, the system has strong nonlinear vibration. This vibration will have a great impact on the performance and reliability of automotive transmission system, such as the impact of noise, resulting in increased transmission errors, etc. A large number of researchers have studied the nonlinear dynamics of a single pair of gears. Sun Tao[4] studied the frequency of a nonlinear vibration of system by using harmonic balance and discussed the progress of period doubling bifurcation in numerical methods. Cui Yahui[5] established the time-varying dynamics model of gear-rotor coupling systems, including the impact of the meshing stiffness and tooth backlash. However, there are few studies on the coupling problem of multi-pairs of gears. In order to reveal characters of the nonlinear gears system with the coupling impact, the research was focused on the electric vehicle with an AMT with 2 forward gears. The five dimension degree model of automated mechanical transmission has been built and compared with the model which neglects the coupling impact.

\section{Model of the Gear System}

Figure 1 shows the simplified schematic views of transmission of the car. Figure 2 is the nonlinear dynamic model of a single pair of gears. We assumed $\theta, \theta^{\prime}, \theta^{\prime \prime}$ stand for vibration angular displacement, vibration angular rate and vibration angular acceleration. $\omega, \omega^{\prime}$ stand for angular rate, angular acceleration, relations $\omega_{p}=i \omega_{g}, \omega_{p}^{\prime}=i \omega_{g}^{\prime}$ is satisfied. ${ }^{r}$ and $r_{g}$ stand for base circle of driving gear and driven gear. ${ }^{c_{m}}, k_{h}$ and ${ }^{e_{(t)}}$ stand for damping coefficient, meshing stiffness and static transmission error. $k_{1}$ and $k_{2}$ stand for supporting stiffness of the two shafts.

\footnotetext{
${ }^{1}$ This work is supported by International Science \& Technology Cooperation Program of China (2014DFG70840)

* Corresponding Author
} 


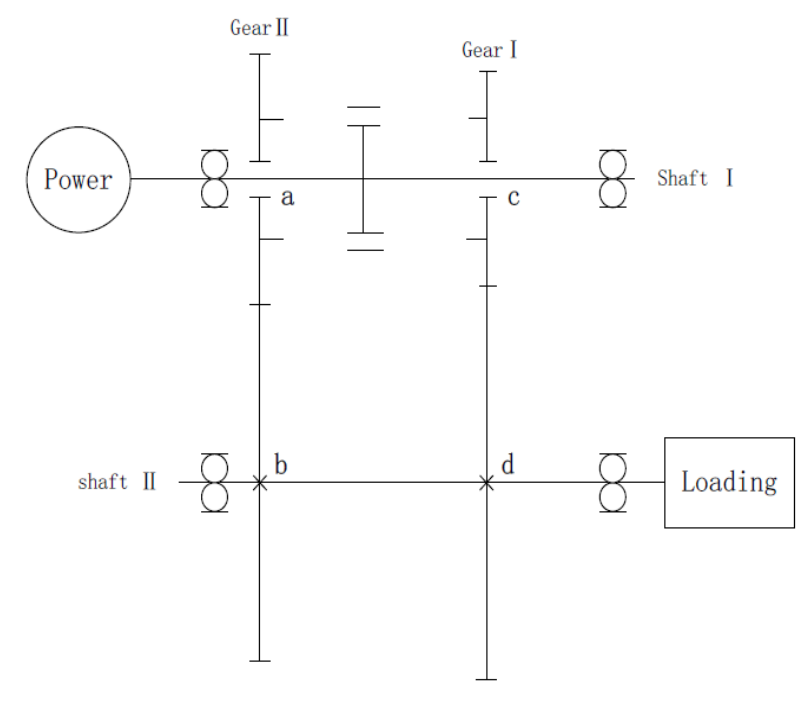

Fig.1 Simplified Schematic Views of Transmission

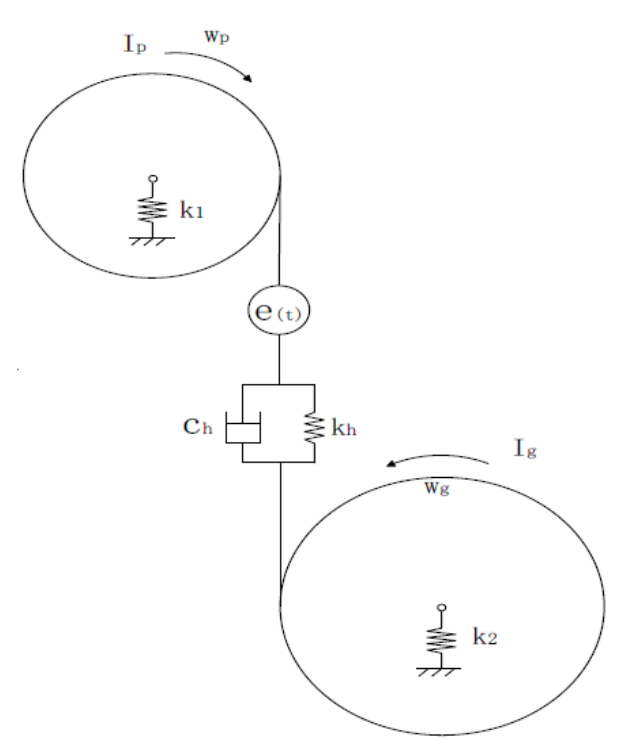

Fig. 2 Model of A Single Pair of Gears

The dynamic equations of gear system are accomplished:

$$
\begin{aligned}
& m_{p}\left(y_{p}\right)^{\prime \prime}+k_{1} y_{p}+c_{h}\left(r_{p} \theta_{p}+\mathrm{y}_{p}-r_{g} \theta_{g}-y_{g}-e\right)^{\prime}+k_{h}(t) f_{h}\left(r_{p} \theta_{p}+\mathrm{y}_{p}-r_{g} \theta_{g}-y_{g}-e\right)=0 \\
& m_{g}\left(y_{g}\right)^{\prime \prime}+k_{2} y_{g}-c_{h}\left(r_{p} \theta_{p}+\mathrm{y}_{p}-r_{g} \theta_{g}-y_{g}-e\right)^{\prime}-k_{h}(t) f_{h}\left(r_{p} \theta_{p}+\mathrm{y}_{p}-r_{g} \theta_{g}-y_{g}-e\right)=0 \\
& I_{p}\left(\theta_{p}\right)^{\prime \prime}+r_{p} c_{h}\left(r_{p} \theta_{p}+\mathrm{y}_{p}-r_{g} \theta_{g}-y_{g}-e\right)^{\prime}+r_{p} k_{h}(t) f_{h}\left(r_{p} \theta_{p}+\mathrm{y}_{p}-r_{g} \theta_{g}-y_{g}-e\right)=T_{p} \\
& I_{p}\left(\theta_{p}\right)^{\prime \prime}-r_{p} c_{h}\left(r_{p} \theta_{p}+\mathrm{y}_{p}-r_{g} \theta_{g}-y_{g}-e\right)^{\prime}-r_{p} k_{h}(t) f_{h}\left(r_{p} \theta_{p}+\mathrm{y}_{p}-r_{g} \theta_{g}-y_{g}-e\right)=-T_{g}
\end{aligned}
$$

If we assumed $I_{p}=m_{1} r_{p}^{2}, I_{\mathrm{g}}=m_{2} r_{g}^{2}, y_{1}=r_{p} \theta_{p}, y_{2}=r_{g} \theta_{g}$, the equations can be changed as follow:

$$
\begin{aligned}
& m_{p}\left(y_{p}\right)^{\prime \prime}+k_{1} y_{p}+c_{h}\left(y_{1}+y_{p}-y_{2}-y_{g}-e\right)^{\prime}+k_{h}(t) f_{h}\left(y_{1}+y_{p}-y_{2}-y_{g}-e\right)=0 \\
& m_{g}\left(y_{g}\right)^{\prime \prime}+k_{2} y_{g-} c_{h}\left(y_{1}+y_{p}-y_{2}-y_{g}-e\right)^{\prime}-k_{h}(t) f_{h}\left(y_{1}+y_{p}-y_{2}-y_{g}-e\right)=0 \\
& m_{1}\left(y_{1}\right)^{\prime \prime}+c_{h}\left(y_{1}+y_{p}-y_{2}-y_{g}-e\right)^{\prime}+k_{h}(t) f_{h}\left(y_{1}+y_{p}-y_{2}-y_{g}-e\right)=F_{m} \\
& m_{2}\left(y_{2}\right)^{\prime \prime}-c_{h}\left(y_{1}+y_{p}-y_{2}-y_{g}-e\right)^{\prime}-k_{h}(t) f_{h}\left(y_{1}+y_{p}-y_{2}-y_{g}-e\right)=-F_{m}
\end{aligned}
$$

Time-variant meshing stiffness and static transmission error is a periodic function of mesh frequency. As the contact ratio of the spiral gear is larger, the fluctuation of stiffness is small. Time-variant meshing stiffness and static transmission error is expressed by a first harmonic frequency:

$$
\begin{gathered}
k_{h}(t)=k_{m}+k_{a} \cos \omega_{h} t \\
e(t)=e \sin \omega_{h} t \\
\omega_{h}=z_{p} \omega_{p}=z_{g} \omega_{g}
\end{gathered}
$$

The tooth backlash can be described as follow:

$$
f_{h}(x)=\left\{\begin{array}{cc}
x-b & x>b \\
0 & -b<x<b \\
x+b & x<-b
\end{array}\right.
$$




\section{Model of the Gear System}

The first gear meshes as the second gear idles, thus the torsional vibrations are not occurred between the two driven gears. So we consider that the vibration angular rate and the angular rate of the two driven gears are identical. In the process of the torque transmitting, the engaging force will not only generate deflection on not only the points $c$ and $d$, but also a and $b$, because of the deformation of the whole draft, which can be calculated through mode superposition method. The draft can be regarded as simple-supported beam, of which the natural frequency and the stress modal function can be described as:

$$
\begin{aligned}
& \omega_{r}=(r \pi)^{2} \sqrt{\frac{E I}{m L^{4}}}, r=1,2,3 \ldots \\
& Y_{r}(x)=\sin \frac{r \pi x}{L}, r=1,2,3 \ldots
\end{aligned}
$$

The equation of deflection curve is

$$
y(x, t)=\sum_{r=1}^{\infty} Y_{r}(x) \eta_{r}(t)
$$

We assume that a time-varying concentrated load Fsin $\omega$ t is applied on the point $\mathrm{c}$. When the excitation frequency is far lower than The natural frequency of the system, the responder function can be changed into

$$
y(x, t)=\frac{2 F L^{3}}{E I \pi^{4}} \sin \omega t \sum_{r=1}^{\infty} \frac{1}{r^{4}} \sin \frac{r \pi c}{L} \sin \frac{r \pi x}{L}
$$

And the deflection of the point $\mathrm{c}$ is

$$
y(c, t)=\frac{2 F L^{3}}{E I \pi^{4}} \sin \omega t \sum_{r=1}^{\infty} \frac{1}{r^{4}}\left(\sin \frac{r \pi c}{L}\right)^{2}
$$

At the same time the deflection of the point a is

$$
y(a, t)=\frac{2 F L^{3}}{E I \pi^{4}} \sin \omega t \sum_{r=1}^{\infty} \frac{1}{r^{4}} \sin \frac{r \pi c}{L} \sin \frac{r \pi a}{L}
$$

Subtracting the two expressions and we can see the ratio is a constant. The constant is independent of the time and the excitation frequency, and related to the position of the points. The meshing forces in finite time satisfy Dirichlet conditions, so it can be converted directly into Fourier series. Hypothesis based on linear elasticity and small deformation of the shaft, the principle of superposition is satisfied. Thus, the ratio of the deflections should be a constant under the effect of the applied time-varying meshing force. According to the positions of the two points, sufficient accuracy can be satisfied by summing up the first four series. The ratio is about 0.74 .According to expressions (3), the coupling equations are defined.

$$
\begin{aligned}
& m_{\mathrm{I} p}\left(y_{\mathrm{II}}\right)^{\prime \prime}+k_{\mathrm{I} 1} y_{\mathrm{I} p}+c_{h}\left(y_{\mathrm{II}}+\mathrm{y}_{\mathrm{I} p}-y_{\mathrm{I} 2}-y_{\mathrm{Ig}}-e_{\mathrm{I}}\right)^{\prime}+k_{\mathrm{IL}}(t) f_{h}\left(y_{\mathrm{II}}+\mathrm{y}_{\mathrm{I} p}-y_{\mathrm{I} 2}-y_{\mathrm{II}}-e\right)=0 \\
& m_{\mathrm{Ig}}\left(y_{\mathrm{Ig}}\right)^{\prime \prime}+k_{\mathrm{I} 2} y_{\mathrm{Ig}}-c_{h}\left(y_{\mathrm{II}}+\mathrm{y}_{\mathrm{I} p}-y_{\mathrm{I} 2}-y_{\mathrm{Ig}}-e_{\mathrm{I}}\right)^{\prime}-k_{\mathrm{I} h}(t) f_{h}\left(y_{\mathrm{I} 1}+\mathrm{y}_{\mathrm{I} p}-y_{\mathrm{I} 2}-y_{\mathrm{Ig}}-e\right)=0 \\
& m_{\mathrm{II}}\left(y_{\mathrm{II}}\right)^{\prime \prime}+c_{h}\left(y_{\mathrm{II}}+\mathrm{y}_{\mathrm{I} p}-y_{\mathrm{I} 2}-y_{\mathrm{Ig}}-e_{\mathrm{I}}\right)^{\prime}+k_{\mathrm{II}}(t) f_{h}\left(y_{\mathrm{II}}+\mathrm{y}_{\mathrm{I} p}-y_{\mathrm{I} 2}-y_{\mathrm{Ig}}-e\right)=F_{\mathrm{II}} \\
& \left(m_{\mathrm{I} 2}+I_{\mathrm{IIg}} / R_{\mathrm{Ig}}^{2}\right)\left(y_{\mathrm{I} 2}\right)^{\prime \prime}-c_{h}\left(y_{\mathrm{I} 1}+\mathrm{y}_{\mathrm{I} p}-y_{\mathrm{I} 2}-y_{\mathrm{Ig}}-e_{\mathrm{I}}\right)^{\prime}-k_{\mathrm{I} h}(t) f_{h}\left(y_{\mathrm{I} 1}+\mathrm{y}_{\mathrm{I} p}-y_{\mathrm{I} 2}-y_{\mathrm{Ig}}-e\right)=-F_{\mathrm{I} m} \\
& m_{\mathrm{II}}\left(y_{\mathrm{III}}\right)^{\prime \prime}+c_{h}\left(y_{\mathrm{II} 1}+0.74 \mathrm{y}_{\mathrm{I} p}-0.875 y_{\mathrm{I} 2}-0.74 y_{\mathrm{Ig}}-e_{\mathrm{II}}\right)^{\prime} \\
& +k_{\mathrm{II} h}(t) f_{h}\left(y_{\mathrm{II} 1}+0.74 \mathrm{y}_{\mathrm{I} p}-0.875 y_{\mathrm{I} 2}-0.74 y_{\mathrm{Ig}}-e_{\mathrm{II}}\right)=0
\end{aligned}
$$




\section{Simulation and Analysis}

According to parameter matching of the transmission system, the data are calculated and presented in Table 1.

Tab. 1 Data of the gear system

\begin{tabular}{|c|c|c|c|c|}
\hline \multirow{2}{*}{} & \multicolumn{2}{|c|}{ first pair of gears } & \multicolumn{2}{c|}{ second pair of gears } \\
\cline { 2 - 5 } & driving gear & driven gear & driving gear & driven gear \\
\hline number of gear teeth & 17 & 48 & 23 & 42 \\
\hline lumped mass/kg & 0.32 & 1.98 & 0.52 & 1.49 \\
\hline rotary inertia/kg·m & $2.85^{*} 10^{\wedge}-4$ & $1.532^{*} 10^{\wedge}-2$ & $9.30^{*} 10^{\wedge}-4$ & $9.00^{*} 10^{\wedge}-3$ \\
\hline model number/mm & 2.5 & 2.5 & 2.5 & 2.5 \\
\hline helix angle & $17.08^{\circ}$ & $17.08^{\circ}$ & $17.08^{\circ}$ & $17.08^{\circ}$ \\
\hline
\end{tabular}

The value of damping coefficient is $65 \mathrm{~N} /(\mathrm{m} / \mathrm{s})$ and support stiffness is $4.8^{*} 10^{\wedge} 8 \mathrm{~N} / \mathrm{m}$. The time-variant meshing stiffness can be expressed as $\left(4.64+0.2 \cos w_{h} \mathrm{t}\right)^{*} 10^{\wedge} 8 \mathrm{~N} / \mathrm{m}$. We assume that the time scale $\tau$ equals $100 \cdot t$, and change the equations in dimensionless method. We select the vibration angular displacement $y_{\mathrm{Ig}}$ of driven gear as studied objects. The two different models under different rotate speeds are simulated by using Runge-Kutta Method in Matlab, and the results are compared. We use model a represent the coupling model, which the simulation result is showed in figure (a), and model $b$ represent a single pair of gears, which the result is showed in figure (b).
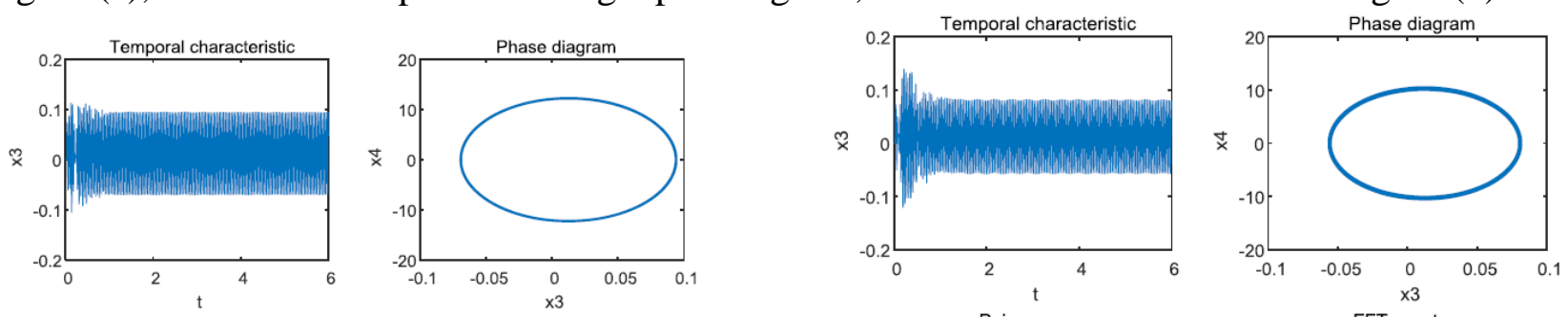
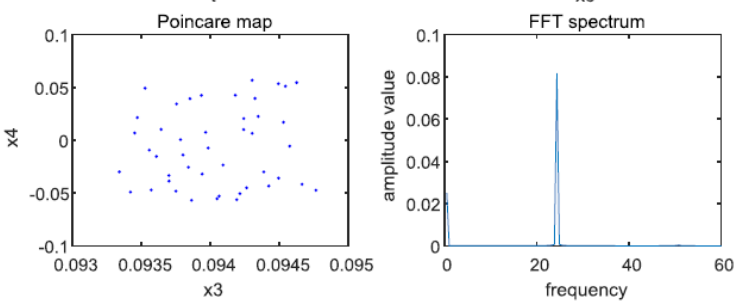

(a)
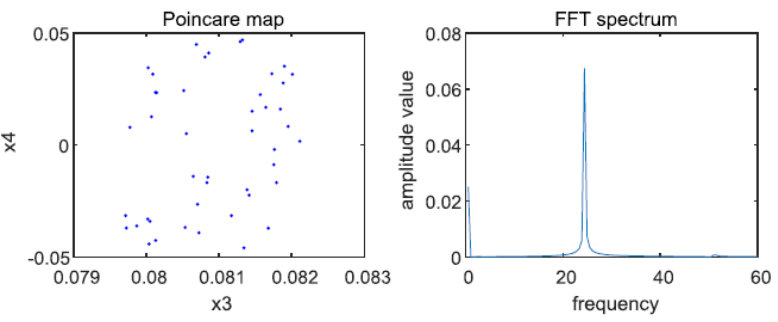

(b)

Fig.3 $\omega=0.24$

As can be seen from figure 3 , when the dimensionless rotate speed is 0.24 , the response of two systems are periodic, which can be inferred from the temporal characteristic and the FFT spectrum. In the FFT spectrum, the frequencies approximately concentrate in $0.1 \mathrm{~Hz}$ and $24 \mathrm{~Hz}$, which are engendered as a superposition of finite sine waves. However, we can see the amplitudes are different in temporal characteristic. The dimensionless amplitude of model a is about 0.8 , while the value is about 0.6 in model $\mathrm{b}$. The difference of the amplitudes between two models can also be revealed in phase diagram and FFT spectrum. The points in Poincare section are finite and the phase diagrams are approximately elliptical. Thus, the response of both models is periodic. 

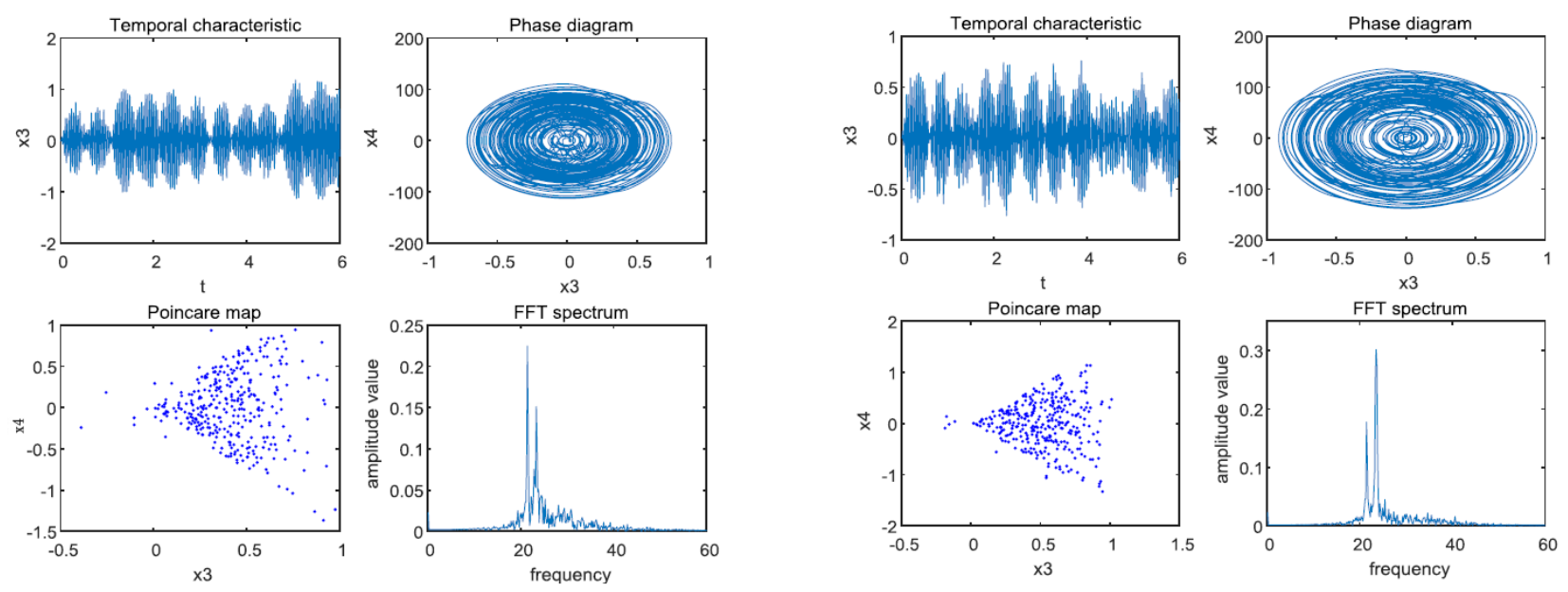

(a)

(b)

Fig. $4 \omega=5$

In the figure 4, it can be seen that both systems is chaotic when the dimensionless rotate speed increases to 5.The temporal characteristic is not periodic any more. The vibration properties of both systems are rambling, which can be seen from the phase diagram and temporal characteristic. Besides, the points in Poincare map are irregular, the number of which is in principle infinite if the period time of simulation is set to infinity. Furthermore, both of the FFT spectra is continuous spectra with certain width. Compared with the former simulation results, the amplitude value increases significantly. Impact is generated at the same time.

\section{Conclusion}

In fact, the coupling between different pair of gears is very common, such as different gears in transmission and intermediate shaft of the two-stage gear reducer, but such a case is usually ignored. The model of system has been built by analyzing coupling impact through mode superposition method under the first gear in a locked-in state condition. The model is simulated in matlab, and the result is compared with the one of the model of a single pair of gears. In conclusion, from the research on the analysis of the simulation, when the response of system is periodic, the amplitude is small. When the response of system is chaotic, the amplitude is apparently augmented and impact is generated. Although the states of the two systems are alike when the dimensionless angular rates are identical, there are significant difference in amplitude-frequency characteristic, which demonstrates the influence of coupling factor to the system and it deserve to be taken into account.

\section{Acknowledgements}

This work is supported by International Science\& Technology Cooperation Program of China (2014DFG70840).

\section{References}

[1] Vaishya M, Houser R. Modeling and analysis of sliding friction in gear dynamics [C]. Proceedings of the 2000ASME Design Engineering Technical Conferences, Baltimore, USA:DETC2000/TG-14430,2000:601-610.

[2] Gang Liu, Robert G Parker. Nonlinear dynamics of idler gear systems [J]. Nonlinear Dynamics, 2008, 53(4): 345-367. 
[3] Lu Jianwei, Liu Mengjun, Chen Lei, Zhao Han. Nonlinear dynamics behavior of gear system with stochastic parameters [J]. Chinese Journal of Construction Machinery, 2009, 20(3): 330 333.

[4] Sun Tao.Nonlinear dynamics of planetary gear train[D].Xi An: North-West Industrial University,2000.

[5] Cui Yahui, Liu Zhansheng, Ye Jianhuai. Vibration Characteristics Analysis of a Gear-Rotor System: Modeling and Simulation [C], Collected Papers of the Eighth National Conference on Dynamics and Control.

[6] Fakher C, Tahar F, Mohamed H. Analytical modeling of spur gear tooth crack and influence on gearmesh stiffness [J], European Journal of Mechanics A/Solids, 2009,10(28): 461-468.

[7] Wei Yongxiang, Chen Jianjun, Wang Minjuan. Dynamic response of torsional vibration of gear-rotor with random Parameters [J]. Journal of Aerospace Power, 2010, 25(11): 2637-2642. 